\title{
The Spleen Promotes the Secretion of CCL2 and Supports an M1 Dominant Phenotype in Hepatic Macrophages During Liver Fibrosis
}

\author{
Liang Lia,b Wei Weja,b Zhenzhen Li ${ }^{a, b}$ Haiyan Chen ${ }^{a} \quad$ Yu Lia,b \\ Wei Jiang ${ }^{a, b}$ Weisan Chen ${ }^{a, c}$ Guangyao Kong ${ }^{a, b}$ Jun Yang ${ }^{a, b}$ \\ Zongfang $\mathrm{Li}^{\mathrm{a}, \mathrm{b}}$
}

aNational \& Local Joint Engineering Research Center of Biodiagnosis and Biotherapy, The Second Affiliated Hospital, Xi'an Jiaotong University, Xi'an, 'The Liver and Spleen Diseases Research Center, Xi'an, 'Department of Biochemistry and Genetics, La Trobe Institute for Molecular Science (LIMS), La Trobe University, Bundoora, Australia

\section{Key Words}

Liver fibrosis $•$ Spleen $\cdot$ Macrophage $\cdot$ Phenotype $\cdot \mathrm{CCL} 2 \cdot$ SOCS3

\begin{abstract}
Background/Aims: Liver fibrosis is a complex process of tissue remodeling in response to injury. Hepatic macrophages have been identified as a key player in this process. As the largest lymphoid organ in the body, the spleen exerts both local and systemic effects on immune cell response. Splenectomy can improve hepatic function during the treatment of liver cirrhosis. However, whether the spleen influences disease progression through the modulation of hepatic macrophages remains unclear. Methods: We examined ex vivo hepatic macrophage responses from splenectomized or sham operated rats and performed splenocyte adoptive transfer studies, in combination with in vivo CCL2 blockade, in splenectomized or sham operated rats. Results: We found that splenectomy reduced fibrosis severity and monocyte/ macrophage infiltration within the injured liver. Splenectomy also reduced secretion of the monocyte chemokine CCL2 by hepatic macrophages. Ex vivo, splenocytes, especially splenic macrophages, promoted CCL2 secretion via upregulation of SOCS3 signaling in hepatic macrophages. Migration of splenic monocytes in response to conditioned medium from hepatic macrophages was inhibited by the blockade of SOCS3-CCL2-CCR2 signaling. Splenectomy also attenuated the establishment of an M1-dominant hepatic macrophage phenotype whilst the adoptive transfer of splenocytes could partly reverse this effect and exacerbate fibrosis. However, CCL2 blockade following adoptive splenocyte transfer restored the protective effects
\end{abstract}

Guangyao Kong, Jun Yang and Zongfang $\mathrm{Li}$
National \& Local Joint Engineering Research Center of Biodiagnosis and Biotherapy The $2^{\text {nd }}$ Aff. Hospital, Xi'an Jiaotong Univ, No.157, Xiwu Road, 710004, Xi'an, (China) E-Mail konggy@xjtu.edu.cn; yangjundr@163.com; Izf2568@xjtu.edu.cn 
of splenectomy. Conclusion: Our study demonstrates that splenic macrophages can promote hepatic macrophage secretion of CCL2, which in turn facilitates monocyte recruitment and the establishment of an M1-dominant hepatic macrophage phenotype, and thus increase the severity of liver fibrosis.

(C) 2018 The Author(s)

Published by S. Karger AG, Basel

\section{Introduction}

Liver fibrosis is characterized by excessive deposition of extracellular matrix in response to persistent injury [1]. The pathogenesis involves a complex interplay between hepatic epithelial cells, pro-inflammatory immune cells, and activated myofibroblasts [2]. Hepatic macrophages are essential for initiating, mediating, and/or perpetuating inflammatory signaling in the fibrotic liver. Two distinct populations of hepatic macrophages are found during liver fibrosis: the residential macrophages termed Kupffer cells, which facilitate pathogen surveillance and metabolite scavenging during homeostasis; and the infiltrating monocyte-derived macrophages, which rapidly expand following an inflammatory insult. Liver injury triggers pattern recognition receptor-mediated Kupffer cell activation and the subsequent release of pro-inflammatory cytokines and chemokines, including CCL2. The chemokine CCL2 facilitates peripheral recruitment of pro-inflammatory monocytes, which then rapidly expand and differentiate into a local pool of monocyte-derived macrophages in the liver [3]. The origins of these infiltrating monocytes are more complex than previously thought as, in addition to the bone marrow, the spleen has recently been proposed as a source of monocytes during liver fibrosis [4-6].

In the fibrotic liver, macrophages exhibit a diverse and plastic spectrum of phenotypes, among which classical (M1) and alternative (M2) represent phenotypic extremes. Functionally, classical (M1) macrophages act as pro-inflammatory effectors of pathogen clearance while alternative (M2) macrophages facilitate inflammation resolution and tissue repair [7]. In the injured liver, macrophages may additionally switch between phenotypes in response to perturbed signals from the hepatic microenvironment [8]. The markers of M1 and M2 macrophages differ between species. In rat, CD68 is a pan marker of monocytes and macrophages and CD86 and CD163 have been widely used to classify M1- and M2-like macrophages respectively $[9,10]$.

As the largest lymphoid organ in the body, the spleen contains a highly elaborate tissue structure and comprises multiple subsets of immune cells, including B and T lymphocytes, macrophages, and dendritic cells, which distribute in the white pulp, marginal zone, and red pulp. These cells play critical roles in the initiation and maintenance of adaptive immunity [11]. The spleen also has a role in blood filtration and immune cell storage. The red pulp harbors a monocyte reservoir capable of mobilizing large numbers of monocytes to participate in inflammatory responses following tissue injury [12]. Splenic innate lymphoid cells can also be mobilized to tissue areas, where they affect disease progression $[13,14]$. Thus, the spleen plays important roles in both local and systemic immunity.

Given the anatomical proximity between the spleen and liver via the portal vein as well as the frequent involvement of splenic abnormalities in liver diseases, a "liver-spleen axis" has been proposed $[15,16]$. Clinically, liver cirrhosis is often accompanied by splenomegaly and hypersplenism. Numerous studies have suggested that the spleen may promote the progression of liver cirrhosis as splenectomy can partly improve liver function [17-19]. Using a thioacetamide (TAA)-induced rat model of liver cirrhosis, Akahoshi et al. demonstrated splenic red pulp macrophages as a potential source of transforming growth factor beta 1 (TGF- $\beta 1$ ) driving hepatic fibrogenesis [20]. They further showed that splenectomy led to decreased serum TGF- $\beta 1$ levels, decreased liver fibrosis, and improved liver regeneration. In addition, in a series of studies our group found that splenic macrophages in patients with cirrhosis are hyperactivated due to elevated nuclear factor-kappa B (NF-kB) signaling and subsequently exacerbate cirrhosis-associated hypersplenism through the release of proinflammatory and pro-fibrogenic factors such as IL-1 $\beta$, IFN- $\gamma$, TNF- $\alpha$, and TGF- $\beta 1$ [21-25]. 


\section{Cellular Physiology Cell Physiol Biochem 2018;51:557-574

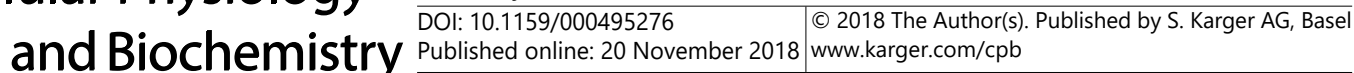

In sum, these studies point to a role for splenic macrophage-derived soluble factors in the progression of liver cirrhosis. Recently, Yada et al. reported that splenectomy significantly increased the proportion of alternative Ly- $6 \mathrm{C}^{\text {low }}$ hepatic macrophages in a TAA-induced murine model of liver cirrhosis [26], further suggesting an influence of the spleen on hepatic macrophage phenotypes. However, precise mechanisms underlying this splenic influence on hepatic macrophage phenotypes during liver fibrosis remain to be established.

In this study, we used a carbon tetrachloride $\left(\mathrm{CCl}_{4}\right)$-induced liver fibrosis model to investigate the impact of splenocytes on hepatic macrophage behavior and liver fibrosis progression. Using splenectomy and splenocyte adoptive transfer techniques, in combination with the blockade of CCL2 or CCR2, we found that splenic macrophages distally promoted the secretion of CCL2 in hepatic macrophages, which in turn facilitated monocyte recruitment and the establishment of an M1-dominant hepatic macrophage phenotype driving liver fibrosis.

\section{Materials and Methods}

\section{Animals and liver fibrosis model}

In this study, we used 6- to 8-week-old male wild-type and GFP transgenic Sprague-Dawley rats purchased from the Laboratory Animal Center of Xi'an Jiaotong University (Xi'an, China) and Cyagen Biosciences Inc. (Guangzhou, China) respectively. Liver fibrosis was induced by intraperitoneal injection of $\mathrm{CCl}_{4}$ (reconstituted in olive oil at a ratio of $1: 1$ and administered at a dose of $1 \mu \mathrm{L} / \mathrm{g}$ body weight) twiceweekly for 6 consecutive weeks [27]. All animal study protocols were approved by the Animal Care and Use Committee of Xi'an Jiaotong University and in accordance with the recommendations of guidelines published by National Institutes of Health, China.

\section{Splenectomy}

Splenectomy was performed as previously described [26]. Splenectomy or sham surgery was performed in rats 4 weeks after induction of liver fibrosis. Following surgery, rats were administered $\mathrm{CCl}_{4}$ for another 2 weeks and euthanized at 6 weeks post-induction of liver fibrosis.

\section{Hematoxylin-eosin, Masson trichrome, and Sirius red staining}

Hematoxylin-eosin, Masson trichrome, and Sirius red staining were performed to assess injury and collagen matrix deposition as previously described [28]. Pictures were obtained from more than five fields per rat using a polarized light filter and quantified by Image J software (National Institutes of Health, Bethesda, MD). At least 5 rats were studied per treatment group.

\section{Immunohistochemistry and immunofluorescence}

For immunohistochemistry, formalin-fixed paraffin-embedded tissue slices ( $5 \mu \mathrm{m})$ were deparaffinized and rehydrated. Antigen retrieval was carried out using citrate buffer. Endogenous peroxidase was quenched with $3 \%$ hydrogen peroxide; $5 \%$ serum was used for blocking. Primary antibodies were diluted in blocking solution at 1:200 for CD68 (ab955, Abcam Cambridge, United Kingdom), 1:200 for CD86 (ab213044, Abcam), 1:200 for CD163 (ab182422, Abcam), 1:100 for CCR2 (ab227015, Abcam), and 1:100 for CCL2 (ab25124, Abcam), and incubated at $4^{\circ} \mathrm{C}$ overnight. Secondary antibody was then added for $1 \mathrm{~h}$ at room temperature. ABC reagent was applied for 30 min followed by the DAB reagent for signal detection. Slides were counterstained with hematoxylin and cover-slipped. Results were expressed as the percentage of positive staining per field and quantified from at least 5 fields per section from at least 5 rats per treatment group.

Immunofluorescence was performed on liver cryosections $(5 \mu \mathrm{m})$. Sections were fixed in ice-cold $100 \%$ acetone and blocked with $10 \%$ serum. Primary antibodies were diluted in blocking solution at 1:50 for SOCS3) (ab14939, Abcam), 1:100 for CD68 (ab125212, Abcam), and 1:100 for GFP (ab183734, Abcam), and incubated at $4^{\circ} \mathrm{C}$ overnight. Goat anti-mouse AF594 or goat anti-rabbit AF488 secondary antibodies were diluted at 1:500 or 1:200 in PBS/1\% bovine serum albumin, respectively, and added for $1 \mathrm{~h}$ at $37^{\circ} \mathrm{C}$. 


\section{Cellular Physiology Cell Physiol Biochem 2018;51:557-574 \begin{tabular}{ll|l} 
and Biochemistry Published online: 20 November 2018 & $\begin{array}{l}\text { (c) } 2018 \text { The Author(s). Published by S. Karger AG, Basel } \\
\text { www.karger.com/cpb }\end{array}$ \\
\hline
\end{tabular} \\ Li et al.: The Spleen and Liver Fibrosis}

Slides were then mounted using ProLong ${ }^{\circledR}$ Gold anti-fade reagent with DAPI and imaged using a Nikon $90 \mathrm{i}$ upright wide-field microscope equipped for epifluorescence. Results were expressed as the number of positive cells per field and quantified from a total of at least 10 fields per section. At least 5 rats per treatment group were examined. Isotype controls are shown in Supplementary Fig. 1 (For all supplemental material see www.karger.com/10.1159/000495276/).

Enzyme-linked immunosorbent assay (ELISA)

ELISA was performed on liver homogenates or the supernatants of cultured macrophages using the rat MCP-1 ELISA Kit (NeoBioscience, China) according to the manufacturer's instructions.

\section{Primary cell isolation}

Hepatic macrophages were isolated from rat livers as previously described [29]. In brief, the liver was perfused with Hank's balanced salt solution, then digested with a solution containing $0.4 \%$ protease (SigmaAldrich, St. Louis, MO) and $0.01 \%$ collagenase (Sigma-Aldrich). The suspension was filtered through a $70 \mu \mathrm{m}$ mesh (BD Falcon cell strainer; BD Biosciences, Franklin Lakes, NJ) and centrifuged at $50 \mathrm{~g}$ for $3 \mathrm{~min}$. Pelleted hepatocytes were collected and the supernatant containing non-parenchymal cells was collected for either flow cytometry or further separation by centrifugation in a discontinuous density gradient of $8.2 \%$ and $15.6 \%$ Nycoden $^{\circledR}$. Kupffer cells in the resulting lower layer were further purified by selective plating [30]. Cell viability was $>90 \%$ as determined by trypan blue staining.

For isolation of splenic leukocytes, the spleen was minced and digested with a solution containing $0.4 \%$ collagenase and then filtered through a $40-\mu \mathrm{m}$ mesh to obtain single-cell suspensions as previously described [12]. Splenic leukocytes were washed twice in PBS by centrifugation at $450 \mathrm{~g}$ at $4^{\circ} \mathrm{C}$ and resuspended in PBS for transfer studies or seeded and cultured in Dulbecco's modified Eagle's medium (DMEM) containing 10\% FBS for cell adherence studies. Splenic macrophages were identified by flow cytometry after staining with anti-CD68, as previously described [25], and using India ink phagocytosis studies. Cell viability was $>90 \%$ as determined by trypan blue staining. The purity of splenic macrophages was $>90 \%$ as determined by flow cytometry (Supplementary Fig. 2).

To harvest single-cell suspensions from the lung and kidney, organs were minced and digested with $0.01 \%$ collagenase and $0.5 \mathrm{mg} / \mathrm{mL}$ DNase (Sigma-Aldrich) for $1 \mathrm{~h}$ at $37^{\circ} \mathrm{C}$ under continuous rotation. Red blood cells (RBCs) were lysed using RBC lysis buffer for $5 \mathrm{~min}$ on ice. Cells were then washed twice with PBS.

To harvest single-cell suspensions from bone marrow, cells were flushed out from femur and tibia with PBS containing 2\% fetal calf serum using a $25 \mathrm{G}$ needle. RBCs were lysed using RBC lysis buffer for 5 min on ice. Cells were filtered through a 40- $\mu \mathrm{m}$ mesh and resuspended in PBS for transfer studies. Bone marrow macrophages were obtained by selective plating. The purity of macrophages was $>90 \%$ as determined by flow cytometry.

\section{Flow cytometric analysis}

Cells were pre-incubated with rat serum (diluted 1:100 in PBS) for $10 \mathrm{~min}$ at $4^{\circ} \mathrm{C}$ to minimize nonspecific antibody binding. Cells were then suspended in FACS buffer (PBS containing 2\% FCS and 0.05\% $\mathrm{NaN}_{2}$ ) and incubated with primary antibodies against the cell-surface markers: anti-CD45 (OX-1; BD Pharmingen $^{\mathrm{TM}}$ ), anti-CD86 (24F, BD Pharmingen ${ }^{\mathrm{TM}}$ ), anti-CD163 (ED2, Bio-Rad), or anti-CCR2 (890231, R\&D SYSTEMS) for $30 \mathrm{~min}$ at $4^{\circ} \mathrm{C}$. 7-AAD was used to differentiate between live and dead cells. For intracellular staining to determine CD68 levels, cells were fixed after surface staining and then permeabilized using $0.3 \%$ Triton X-100 for $30 \mathrm{~min}$, before anti-CD68 staining (ED1, Bio-Rad) for $30 \mathrm{~min}$. Cell counts were determined using a BD FACS Canto II flow cytometer (BD Biosciences) and analyzed with FlowJo software (FlowJo, LLC, Ashland, OR). The gating strategy and control experiments are shown in Supplementary Fig. 3. The antibodies are shown in Supplementary table 1.

\section{Adoptive transfer}

Isolated splenocytes or bone marrow cells from wild-type or GFP transgenic rats were resuspended in PBS. Approximately $4.5 \times 10^{7}$ cells were intravenously injected per rat for adoptive transfer studies. 


\section{Cellular Physiology Cell Physiol Biochem 2018;51:557-574 and Biochemistry \begin{tabular}{l|l} 
DOI: 10.1159/000495276 2018 The Author(s). Published by S. Karger AG, Basel \\
Publishedon
\end{tabular}

\section{Quantitative reverse transcription PCR ( $q P C R)$}

Total RNA was extracted with TRIzol (Takara, Japan) and then reverse transcribed to complementary DNA using a Primescript ${ }^{\mathrm{TM}}$ RT reagent kit (Takara). qPCR was then performed using SYBR Premix Ex Taq II and an ABI7500 fast instrument (ABI Life technologies). The relative abundance of the target genes was determined by the comparative cycle threshold $\mathrm{Ct}$ method $\left(2^{-\Delta \Delta \mathrm{Ct}}\right)$ and normalized to $\beta$-actin levels. The primers are shown in Supplementary table 2.

\section{Small interfering RNA (siRNA)}

Cells were transfected with either $100 \mathrm{nM}$ scrambled or SOCS3 siRNA duplex, using. Lipofectamine ${ }^{3000}$ (Thermo Scientific, Waltham, MA) according to the manufacturer's instructions. Medium was replaced at $12 \mathrm{~h}$ after transfection and cells were rested for another $12 \mathrm{~h}$. The siRNA targeting sequences: CCTGGACCAGTATGATGCTCCACTT.

\section{Western blotting}

Whole-cell lysates were obtained using lysis buffer (50 mM Tris-HCl, pH 7.4; $1 \%$ Nonidet P-40; 0.25\% sodium deoxycholate; $150 \mathrm{mM} \mathrm{NaCl} ; 1 \mathrm{mM}$ EDTA; $1 \mathrm{mM}$ PMSF; $1 \mu \mathrm{g} / \mathrm{mL}$ aprotinin, leupeptin, pepstatin). Protein concentration was measured using the BCA protein assay kit (HEART, China). Equal amounts of protein were then loaded onto an SDS-PAGE gel, transferred to a polyvinylidene fluoride membrane, and incubated overnight with primary antibodies (SOCS3, ab14939; CCR2, ab227015; both Abcam, diluted 1:1000). Horseradish peroxidase-conjugated secondary antibody (Santa Cruz Biotechnologies, diluted at $1: 3000$ ) was then added. $\beta$-actin was used as an internal control. Proteins of interest were detected using enhanced chemiluminescence (Amersham, Arlington Heights, IL) with the Bio-Rad imaging system.

\section{Conditioned medium}

Conditioned medium from isolated primary cells was generated as previously described [31]. In brief, $1 \times 10^{5}$ splenocytes, bone marrow cells, or hepatic macrophages were cultured in $500 \mu \mathrm{L}$ serum-free DMEM in 24-well plates for $18 \mathrm{~h}$. The supernatants were then collected and centrifuged to remove cell debris. The resulting medium was mixed with an equal volume of fresh medium before addition to cultured cells. After incubation for $6 \mathrm{~h}$, the effects of conditioned medium on cultured cells were assessed.

\section{Transwell migration}

Splenocyte migration was assessed using modified Boyden chambers (Thermo Scientific) loaded onto a 24-well plate. Hepatic macrophage-conditioned medium $(250 \mu \mathrm{L})$ was added to the lower compartment, which was separated from the upper compartment by a gelatin-coated polycarbonate membrane $(13 \mathrm{~mm}$ disk, $8 \mu \mathrm{m}$ ). After $2 \mathrm{~h}$ of serum starvation, $5 \times 10^{4}$ splenocytes were suspended in $0.5 \mathrm{~mL}$ DMEM and seeded in the upper compartment of the Boyden chamber and incubated for $8 \mathrm{~h}$ at $37^{\circ} \mathrm{C}$. The membrane was then fixed with methanol for $30 \mathrm{~min}$ and stained with $0.1 \%$ crystal violet for $20 \mathrm{~min}$. The number of migrated cells was then quantified using a DMI3000B microscope (Leica, Germany).

\section{Ex vivo CCR2 inhibition}

The highly selective CCR2 chemokine receptor antagonist RS504393 (10 mM; Abcam) was used to inhibit CCR2 ex vivo.

Ex vivo and in vivo CCL2 blockade

Rabbit anti-rat CCL2-neutralizing antibody (ab25124, Abcam) was used to block CCL2 ex vivo and in vivo. The concentrations of CCL2-neutralizing antibody were $0.1 \mu \mathrm{g} / \mathrm{g}$ body weight for in vivo studies (administered twice per week by intraperitoneal injections) and $4 \mu \mathrm{g} / \mathrm{mL}$ for ex vivo studies [32].

\section{Statistical analysis}

Data are expressed as the means \pm SEM. Unpaired Student t-test was used for comparison of two groups. To compare values obtained from three or more groups, one or two-way analysis of variance (ANOVA) was used, followed by Tukey's post-hoc test. $P<0.05$ was considered as statistically significant. All analyses were performed using GraphPad Prism 5 (GraphPad Software Inc., San Diego, CA). 


\section{Cellular Physiology and Biochemistry \begin{tabular}{l|l|l} 
DOI: 10.1159/000495276 & $\begin{array}{l}\text { @ } 2018 \text { The Author(s). Published by S. Karger AG, Basel } \\
\text { www.karger.com/cpb }\end{array}$
\end{tabular} Li et al.: The Spleen and Liver Fibrosis}

\section{Results}

\section{Splenectomy ameliorates $\mathrm{CCl}_{4}$-induced liver fibrosis}

To investigate the impact of the spleen on hepatic fibrosis, we utilized a rat model which comprised intraperitoneal injections of $\mathrm{CCl}_{4}$ twice-weekly for 6 weeks. Splenectomy or sham surgery was performed $24 \mathrm{~h}$ after the eighth $\mathrm{CCl}_{4}$ injection and rats were euthanized $72 \mathrm{~h}$ after the last (12th) injection of $\mathrm{CCl}_{4}$. The liver/body mass ratio was analyzed and the livers collected for sectioning. Hepatic collagen deposition was determined by Masson trichrome staining. Repeated $\mathrm{CCl}_{4}$ exposure significantly increased collagen deposition within injured liver tissue and increased liver/body mass ratios in rats (Fig. 1A). Splenectomy significantly ameliorated $\mathrm{CCl}_{4}$-induced hepatic collagen deposition and decreased liver/body mass ratios compared with sham surgery. Liver mRNA levels of the fibrosis-associated genes Acta2 and Col1a1 also decreased following splenectomy compared with sham surgery (Fig. 1B). Since hepatic macrophages can drive the pathogenesis of liver fibrosis, we first investigated the

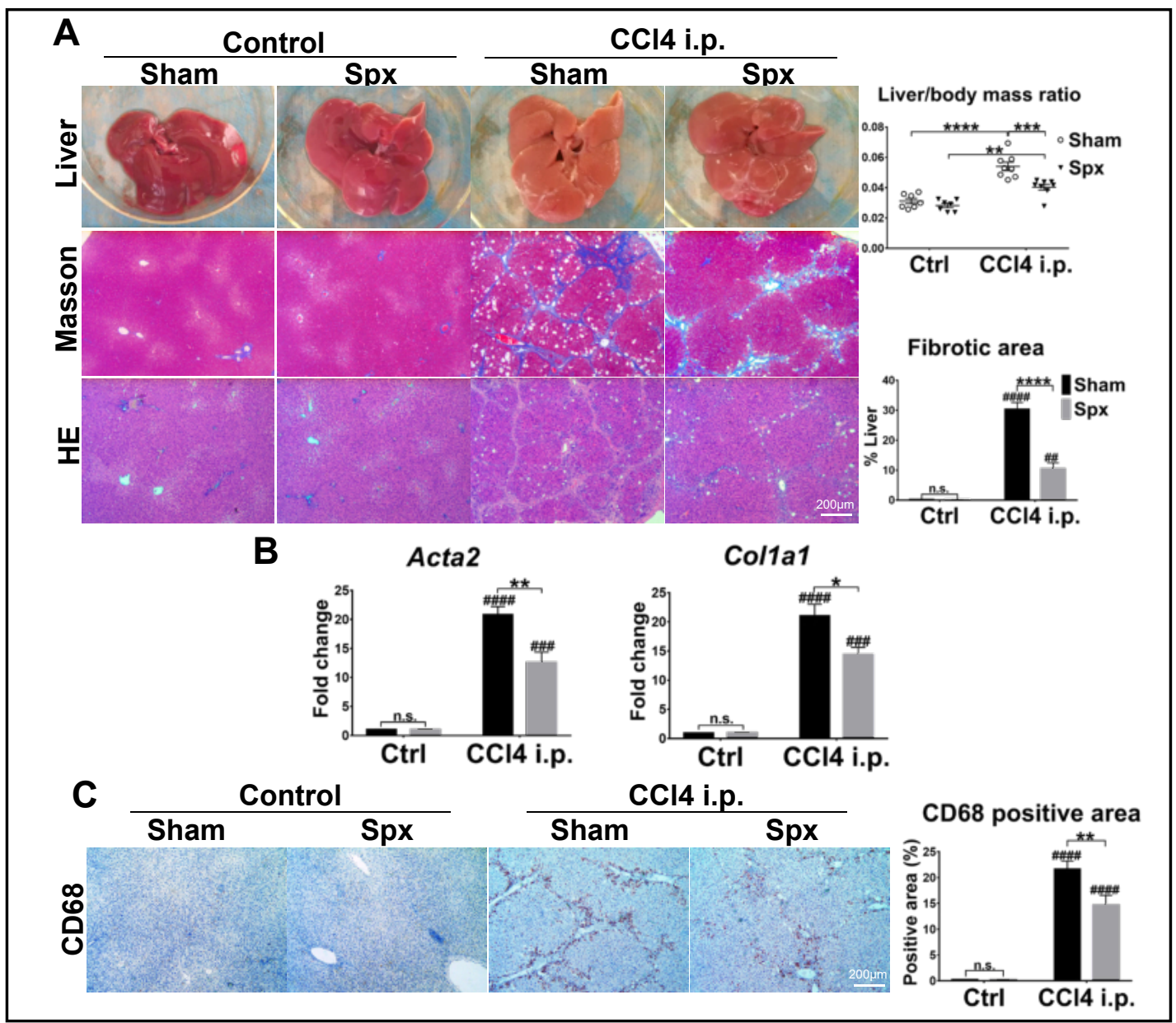

Fig. 1. Splenectomy ameliorates carbon tetrachloride $\left(\mathrm{CCl}_{4}\right)$-induced liver fibrosis. Splenectomy or sham surgery was performed $24 \mathrm{~h}$ after the eighth $\mathrm{CCl}_{4}$ injection, and rats were euthanized $72 \mathrm{~h}$ after the last (12th) $\mathrm{CCl}_{4}$ injection. (A) Liver/body mass ratios were calculated. Liver sections were stained with Masson trichrome or hematoxylin-eosin. (B) Fibrosis-associated genes Acta2 and Col1a1 were measured using qPCR. (C) CD68 immunohistochemical staining quantified in liver sections. Magnification: $\times 50$; scale bar: $200 \mu \mathrm{m}$. Statistics: two-way ANOVA (A: $\mathrm{n}=7$ each, in control Sham or Spx group, $\mathrm{n}=8$ in $\mathrm{CCl}_{4}$ i.p. Sham or Spx group; B,C: $\mathrm{n}=5$ ). ${ }^{*} P<0.05,{ }^{* *} P<0.01,{ }^{* * *} P<0.001,{ }^{* * * *} P<0.0001$, Spx group vs. Sham group. ${ }^{*} P<0.05$,

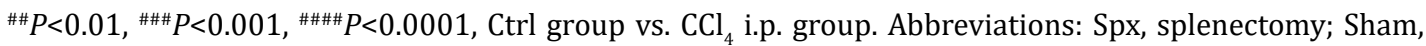
sham operation. 
Fig. 2. Splenectomy decreases hepatic monocyte/macrophage via modulation of CCL2 secretion. Hepatic nonparenchymal cells were isolated. (A) Hepatic monocyte/macrophage proportions were determined using flow cytometry after staining with anti-CD68 and anti-CD45 antibodies. (B) CCL2 mRNA levels were examined using qPCR and protein levels were assayed by ELISA in liver tissues. (C) Primary hepatic cells were isolated and the levels of CCL2 mRNA examined by qPCR. (D) Hepatic macrophages were isolated. CCL2 protein levels in the supernatant of cultured primary hepatic macrophages were measured using ELISA. Statistics: twoway ANOVA (A: $\mathrm{n}=8$; B,C,D: $\mathrm{n}=5$ ). ${ }^{*} P<0.05$,

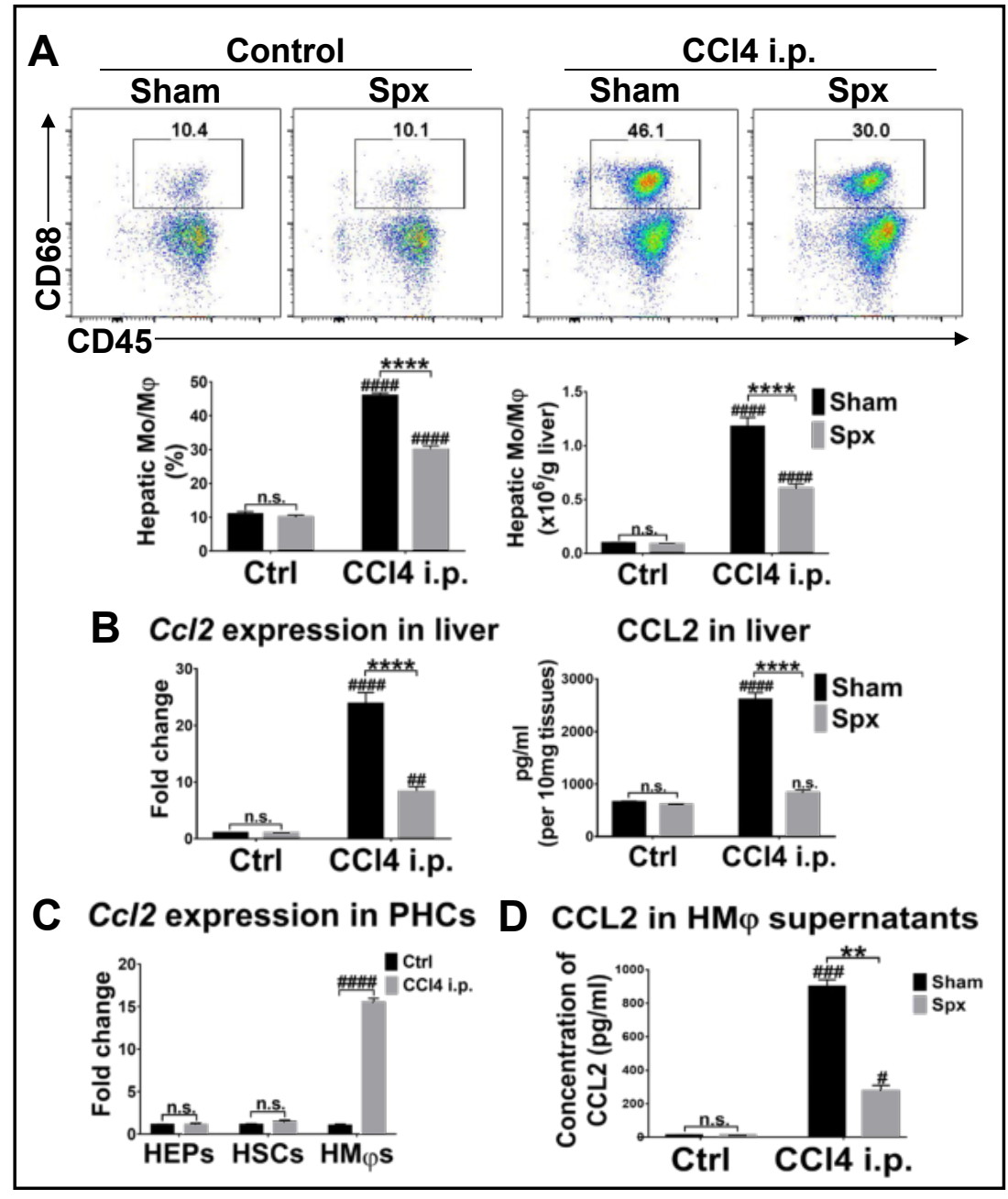

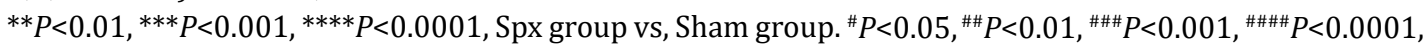
Ctrl group vs. $\mathrm{CCl}_{4}$ i.p. group. Abbreviations: $\mathrm{Mo} / \mathrm{M} \varphi$, monocyte/macrophage; $\mathrm{PHC}$, primary hepatic cell; HEP, hepatocyte; HSC, hepatic stellate cell; HM $\varphi$, hepatic macrophage.

influence of splenectomy on hepatic macrophages by immunohistochemical staining of the monocyte/macrophage marker $\mathrm{CD} 68$. Repeated $\mathrm{CCl}_{4}$ exposure greatly increased the proportion of $\mathrm{CD} 68^{+}$monocytes/macrophages in the liver, and the proportion decreased following splenectomy compared with sham surgery (Fig. 1C), highlighting the link between the spleen and the recruitment and/or distribution of hepatic monocytes/macrophages during liver fibrosis.

Splenectomy decreases hepatic monocyte/macrophage via modulation of CCL2 secretion

To further determine whether splenectomy could alter hepatic monocyte/ macrophage number, we isolated hepatic non-parenchymal cells and analyzed the percentages and absolute numbers of hepatic monocytes/macrophages $\left(\mathrm{CD} 45^{+} \mathrm{CD} 68^{+}\right)$by flow cytometry. The numbers of hepatic monocytes/macrophages were significantly increased in the liver of fibrotic rats compared with healthy controls. Splenectomy significantly attenuated the accumulation of hepatic monocytes/macrophages in fibrotic livers compared with sham surgery (Fig. 2A). In addition, we measured monocytes in peripheral blood and found that splenectomy also decreased the percentages and absolute numbers of circulating monocytes in fibrotic rats (Supplementary Fig. 4). 
Since the expansion of the hepatic macrophage pool is predominantly attributed to peripherally recruited monocytes during liver fibrosis [6], we hypothesized that splenectomy could reduce the recruitment of monocytes into the fibrotic liver. Therefore, we measured the level of several common chemokines reported to participate in liver fibrosis and found that the expression of the monocyte chemokine CCL2 was significantly upregulated in fibrotic compared with healthy livers and downregulated following splenectomy compared with sham surgery (Fig. 2B). Levels of the chemokines CXCL12 and CX3CL1 were also upregulated in liver tissue during fibrosis but unaltered following splenectomy (Supplementary Fig. $5 \mathrm{~A}$ ). To determine whether macrophages were the hepatic source of CCL2, we performed immunofluorescent co-staining of CCL2 and CD68 in liver sections and detected an increase in the number of $\mathrm{CCL} 2^{+} \mathrm{CD} 8^{+}$cells during liver fibrosis (Supplementary Fig. 5B). In addition, we isolated different primary hepatic cell types, measured CCL2 mRNA levels, and found that hepatic macrophages were the major source of CCL2 (Fig. 2C). Ex vivo CCL2 secretion was increased in hepatic macrophages isolated from fibrotic compared with healthy rats and decreased in hepatic macrophages following splenectomy compared with sham surgery (Fig. 2D). Collectively, these results suggest that the spleen and/or splenic signaling increases the recruitment and expansion of monocytes/macrophages during liver fibrosis, most likely through the modulation of CCL2 production by hepatic macrophages.

Splenic macrophages isolated from fibrotic rats promote hepatic macrophage CCL2 secretion via the upregulation of SOCS3 signaling

As the spleen harbors numerous immune cells, we hypothesized that it could affect hepatic macrophage CCL2 secretion through the activity of distal splenic immune cells. First we stimulated primary hepatic macrophages with conditioned medium from splenic immune cells. CCL2 mRNA and protein levels were significantly upregulated in hepatic macrophages exposed to conditioned medium from splenic macrophages isolated from fibrotic compared with healthy rats. Importantly, CCL2 expression was not affected by exposure to conditioned medium collected from non-macrophage splenic cells (Fig. 3A; Supplementary Fig. 6A, B). In addition, we did not detect significant changes in CCL2 expression when hepatic macrophages were cultured in conditioned medium from either total bone marrow cells or purified bone marrow macrophages (Supplementary Fig. 6C). These results suggest that splenic macrophages are selectively capable of upregulating CCL2 secretion in hepatic macrophages during liver fibrosis.

Since CCL2 production is correlated with SOCS3 activity [33], we postulated that hepatic CCL2 expression may be regulated by alterations in SOCS3 signaling. First we examined SOCS3 expression in liver sections through immunofluorescent co-staining of SOCS3 and CD68. The numbers of SOCS3 ${ }^{+} \mathrm{CD} 68^{+}$cells were increased in fibrotic compared with healthy liver sections and decreased following splenectomy but not with sham surgery (Fig. 3B). The highest levels of SOCS3 production from ex vivo cultured hepatic macrophages were found in $\mathrm{CCl}_{4}$-injected and sham-operated rats. (Fig. 3C). To confirm the role of SOCS3 signaling in CCL2 secretion, we stimulated primary hepatic macrophages with conditioned medium from splenic macrophages which were treated with either SOCS3-specific siRNA or scrambled siRNA. siRNA-mediated knockdown of SOCS3 significantly reduced CCL2 upregulation (Fig. 3D; Supplementary Fig. 7). Taken together, these results indicate that splenic macrophages from fibrotic rats can distally promote CCL2 expression via upregulation of SOCS3 signaling in hepatic macrophages.

Blockade of CCL2-CCR2 signaling decreases hepatic macrophage-induced migration of splenic monocytes

To further investigate the role of splenic immune cells on hepatic macrophage CCL2 expression and subsequent monocyte recruitment during hepatic fibrosis, we examined the expression of the chemokine receptor CCR2 in different tissues. As the bone marrow and spleen are established sources of infiltrating monocytes in the fibrotic liver, we investigated CCR2 expression in liver, spleen, and bone marrow cells and found significantly upregulated 


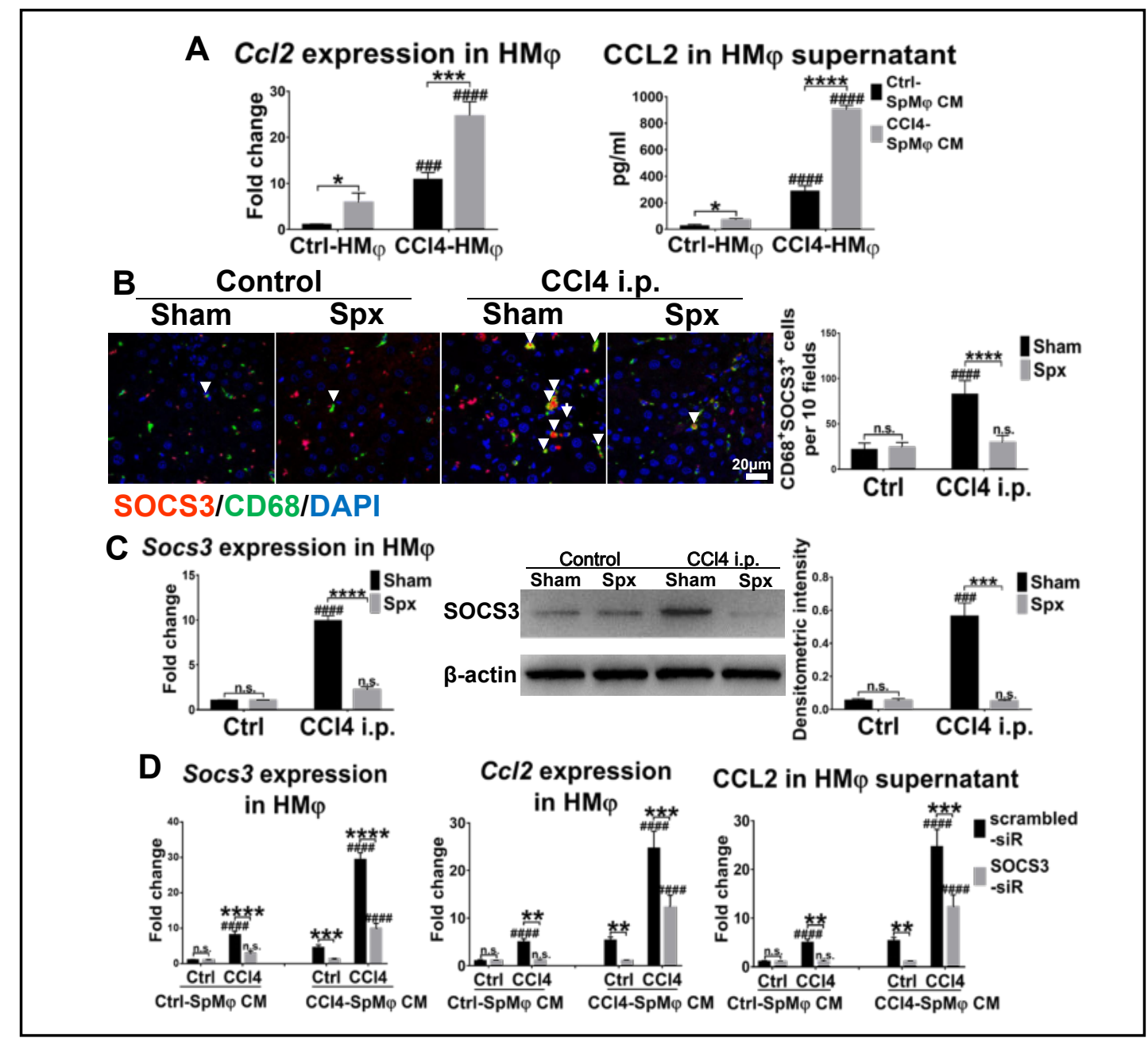

Fig. 3. Splenic macrophages promote hepatic macrophage CCL2 secretion via the upregulation of SOCS3 signaling. (A) Hepatic and splenic macrophages were isolated from fibrotic or healthy control rats and cultured ex vivo. Hepatic macrophages were stimulated with conditioned medium derived from splenic macrophages. After stimulation, CCL2 mRNA and protein levels were measured by qPCR and ELISA, respectively. (B) Livers were sectioned and SOCS3 and CD68 co-staining was examined using immunofluorescence. Magnification: $\times 630$; scale bar: $10 \mu \mathrm{m}$. (C) SOCS3 mRNA and protein levels were measured by qPCR and western blotting, respectively. (D) Hepatic macrophages were stimulated with conditioned medium from splenic macrophages transfected with SOCS3-specific or scrambled siRNA. SOCS3 and CCL2 mRNA levels were determined by qPCR. Statistics: two-way ANOVA (A,C,D: $\mathrm{n}=5$; B: $\mathrm{n}=10$ ). ${ }^{*} P<0.05,{ }^{* *} P<0.01,{ }^{* * *} P<0.001,{ }^{* * * *} P<0.0001$, Ctrl $\mathrm{SpM} \varphi$-CM group vs. $\mathrm{CCl}_{4} \mathrm{SpM} \varphi$-CM group, Spx group vs. Sham group, or SOCS-siR group vs. scrambled

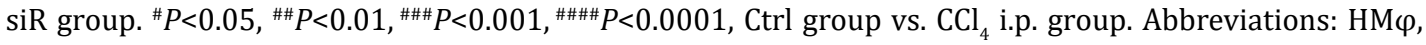
hepatic macrophage; $\operatorname{SpM} \varphi$, splenic macrophage; $\mathrm{CM}$, conditioned medium.

CCR2 expression in both splenic and bone marrow cells during fibrosis (Supplementary Fig. 8A, B). Focusing on splenic cells, we examined splenic cell CCR2 expression by immunohistochemistry and found that CCR2 was mainly expressed in the red pulp of the spleen, which is the site of the splenic monocyte reservoir (Supplementary Fig. 8C). These results were further complemented by flow cytometric studies investigating CCR2 and CD68 splenic cell co-staining. We found that the numbers of $\mathrm{CD} 68^{+} \mathrm{CCR} 2^{+}$splenic cells were significantly increased in fibrotic rats but not in healthy control rats (Supplementary Fig. 8D). Altogether, these results implicated splenic monocytes as a source of CCL2-CCR2-driven monocyte/macrophage infiltration during liver fibrosis. 
Fig. 4. Blockade of CCL2-CCR2 signaling decreases hepatic macrophage-induced migration of splenic monocytes.

Bone marrow cell migration capacity was investigated in response to hepatic macrophageconditioned medium with or without blockade of CCL2CCR2 signaling. (B) Splenocyte migration capacity was investigated in response to hepatic macrophageconditioned medium. Statistics: two-way ANOVA $(\mathrm{n}=5) .{ }^{*} P<0.05$, ${ }^{* *} P<0.01, \quad{ }^{* * *} P<0.001$, ${ }^{* * * *} P<0.0001$, isotype group vs. anti-CCL2 group or solvent group vs. CCR2 antagonist group. $\quad{ }^{*} P<0.05$, $\# P<0.01, \quad \# \# P<0.001$,

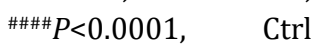
group vs. $\mathrm{CCl}_{4}$ i.p. group. (C) Hepatic macrophages were transfected with

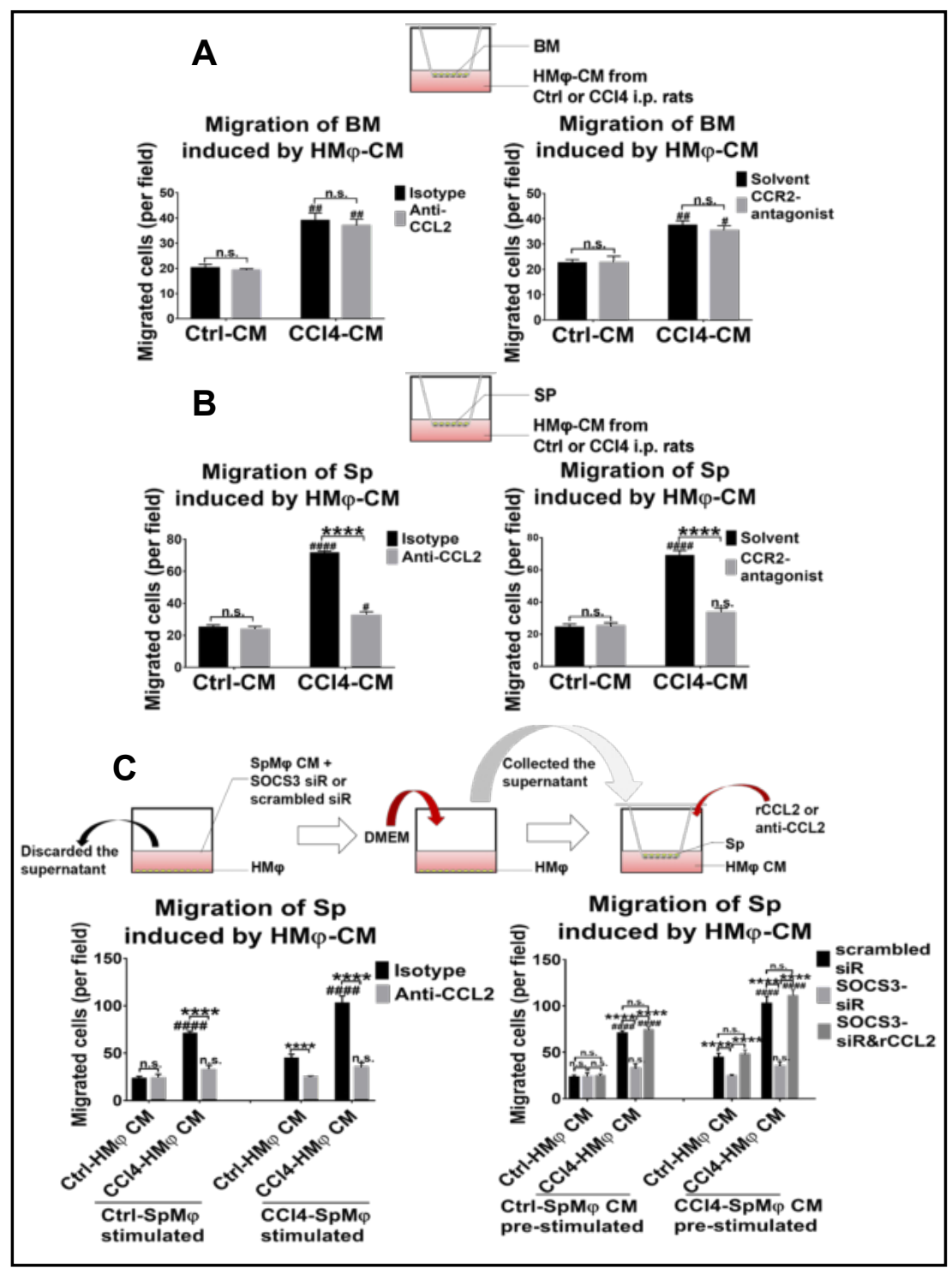
SOCS3-specific or scrambled siRNA and treated with splenic macrophage supernatant. Conditioned medium was then collected. Bone marrow cell or splenocyte migration capacity was investigated in response to the conditioned medium with or without addition of recombinant rat CCL2 (rCCL2). Statistics: unpaired t test $(\mathrm{n}=5) .{ }^{*} P<0.05,{ }^{* *} P<0.01,{ }^{* * *} P<0.001$, ${ }^{* * * *} P<0.0001$, isotype group vs. anti-CCL2 group, or vs.Scrambled siR group, SOCS3-siR group, or SOCS3-

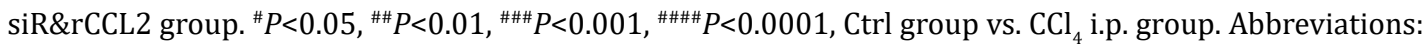
$\mathrm{BM}$, bone marrow cell; Sp, splenocyte; rCCL2, recombinant rat CCL2.

To formally investigate this potential chemotactic axis, we developed a transwell migration assay using splenocytes or bone marrow cells as the migrating cells. Splenocytes or bone marrow cells were incubated with conditioned medium from hepatic macrophages in the Boyden chamber, in the absence or presence of CCL2-blocking antibodies or a CCR2 antagonist. As expected, migration of both splenocytes and bone marrow cells could be induced by conditioned medium from hepatic macrophages. Hepatic macrophageconditioned medium from fibrotic rats induced the greatest degree of migration of splenocytes and bone marrow cells (Supplementary Fig. 9A). Most migratory cells were CD68 ${ }^{+}$as shown by immunofluorescent staining (Supplementary Fig. 9B). Interestingly, addition of the CCL2- 
neutralizing antibody or CCR2 antagonist attenuated splenic cell but not bone marrow cell migration (Fig. 4A, B). Since CCL2 predominantly attracts monocytes, our results indicate that the CCL2-CCR2 axis plays an important role in the migration of splenic but not bone marrow monocytes during liver fibrosis. Furthermore, we found that the chemokine receptor CXCR4 was markedly upregulated in bone marrow cells but not splenocytes from fibrotic compared with healthy control rats (Supplementary Fig. 9C), suggesting that CXCR4 signaling may be particularly important for bone marrow monocyte recruitment during liver fibrosis.

To investigate whether the hepatic macrophage-induced migration of monocytes was affected by splenic macrophages, we used conditioned medium from hepatic macrophages pre-stimulated with splenic macrophage supernatant. We found that the pro-migratory effect of conditioned medium was enhanced when hepatic macrophages were pre-stimulated with splenic macrophage supernatant from fibrotic but not healthy control rats. This enhancement was negated by the blockade of CCL2 or knockdown of SOCS3. Attenuated migration induced by SOCS3 knockdown could be rescued by administration of recombinant rat CCL2, further confirming the role of SOCS3 signaling in CCL2 production (Fig. 4C). Taken together, these data further support a role for the influence of splenic macrophages on SOCS3-dependent CCL2 production in hepatic macrophages during liver fibrosis, and the subsequent CCL2CCR2-mediated recruitment of splenic monocytes into the liver. It is also possible that splenic macrophages may enhance the recruitment of bone marrow-derived cells during liver fibrosis, although not via the CCL2-CCR2 axis (Supplementary Fig. 9D).

Splenectomy reverses the M1-dominant hepatic macrophage phenotype in fibrotic liver

Since the spleen modulated CCL2 production in hepatic macrophages and the subsequent recruitment of splenic monocytes into fibrotic liver, we further investigated whether the spleen could influence the phenotype of hepatic macrophages. Hepatic non-parenchymal cells were isolated and analyzed by flow cytometry for CD68, CD86, and CD163 expression. We combined these markers to define hepatic macrophage phenotypes as being classically (M1-like, CD68 ${ }^{+} \mathrm{CD}^{2} 6^{+}$) or alternatively activated (M2-like, CD68 $\left.{ }^{+} \mathrm{CD} 163^{+}\right)[10,34]$. We found that the proportion of M1-like hepatic macrophages significantly increased whereas the proportion of M2-like macrophages significantly decreased in fibrotic livers compared with healthy controls (Fig. 5A), which was consistent with the results of immunohistochemistry (Supplementary Fig. 10). Overall, the ratio of M1-like to M2-like macrophages was highly elevated in fibrotic livers. Splenectomy significantly reduced the proportions of M1-like macrophages whilst slightly increased the proportions of M2-like macrophages in the liver, producing a lowered M1/M2 ratio (Fig. 5A). We also examined the expression of M1- and M2-associated genes (M1: Nos2, Il-1 $\beta$, Tnf- $\alpha$; M2: Arg1, Il-4, Il-10) in liver tissues by qPCR. Accordingly, M1-associated gene expression levels were upregulated whereas M2-associated gene expression levels were downregulated in fibrotic compared with healthy control livers. This expression pattern was reversed following splenectomy (Fig. 5B). Collectively, our data suggest that the spleen promotes a dominant M1-like hepatic macrophage phenotype in fibrotic rats, and that this process can be significantly reversed by splenectomy.

Adoptive transfer of fibrotic donor splenocytes into splenectomized recipients partially restores the M1-dominant hepatic macrophage phenotype and promotes fibrosis

To confirm that splenocytes from fibrotic rats could alter the phenotype and number of hepatic macrophages, we adoptively transferred $\mathrm{GFP}^{+}$splenocytes $\left(4.5 \times 10^{7}\right.$ cells $\left./ \mathrm{rat}\right)$ isolated from fibrotic donors into $\mathrm{CCl}_{4}$-induced fibrotic recipients that had undergone either splenectomy or sham surgery 3 days previously. The liver, lungs, and kidneys were collected 3 days after splenocyte transfer. We detected $\mathrm{GFP}^{+} \mathrm{CD} 8^{+}$cells mainly in fibrotic liver (Supplementary Fig. 11A) and these cells were mainly found within the fibrotic septa (Supplementary Fig. 11B), directly implicating preferential splenic monocyte/macrophage recruitment and a role in liver fibrogenesis. To determine the effect of splenocytes on the phenotype ofhepaticmacrophages, weadoptively transferred splenocytesintosplenectomised 
Fig. 5. Splenectomy reverses the M1dominant hepatic macrophage phenotype in fibrotic liver. (A) Hepatic nonparenchymal cells were isolated from fibrotic and healthy control rats and analyzed by flow cytometry. The frequencies of M1-like $\left(\mathrm{CD}^{\circ}{ }^{+} \mathrm{CD}^{\circ} 6^{+}\right)$and $\mathrm{M} 2-$ like $\quad\left(\mathrm{CD}^{2} 8^{+} \mathrm{CD} 163^{+}\right)$ hepatic macrophage subpopulations were compared. The ratio of M1 to M2 macrophages was also analyzed. (B) mRNA levels of M1-associated (Nos2, Il-1 $\beta$, Tnf- $\alpha$ ) and M2associated (Arg1, Il-4, Il-10) genes in the liver were determined by qPCR. Statistics: twoway ANOVA (A: $\mathrm{n}=$ 8; $\mathrm{B}: \mathrm{n}=5$ ). ${ }^{*} P<0.05$, ** $P<0.01, \quad * * * P<0.001$, $* * * * P<0.0001$, Spx group vs. Sham group. ${ }^{\#} P<0.05$, \#\# $P<0.01$, \#\# $P<0.001$, \#\#\#\# $P<0.0001$, Ctrl group vs. $\mathrm{CCl}_{4}$ i.p. group.

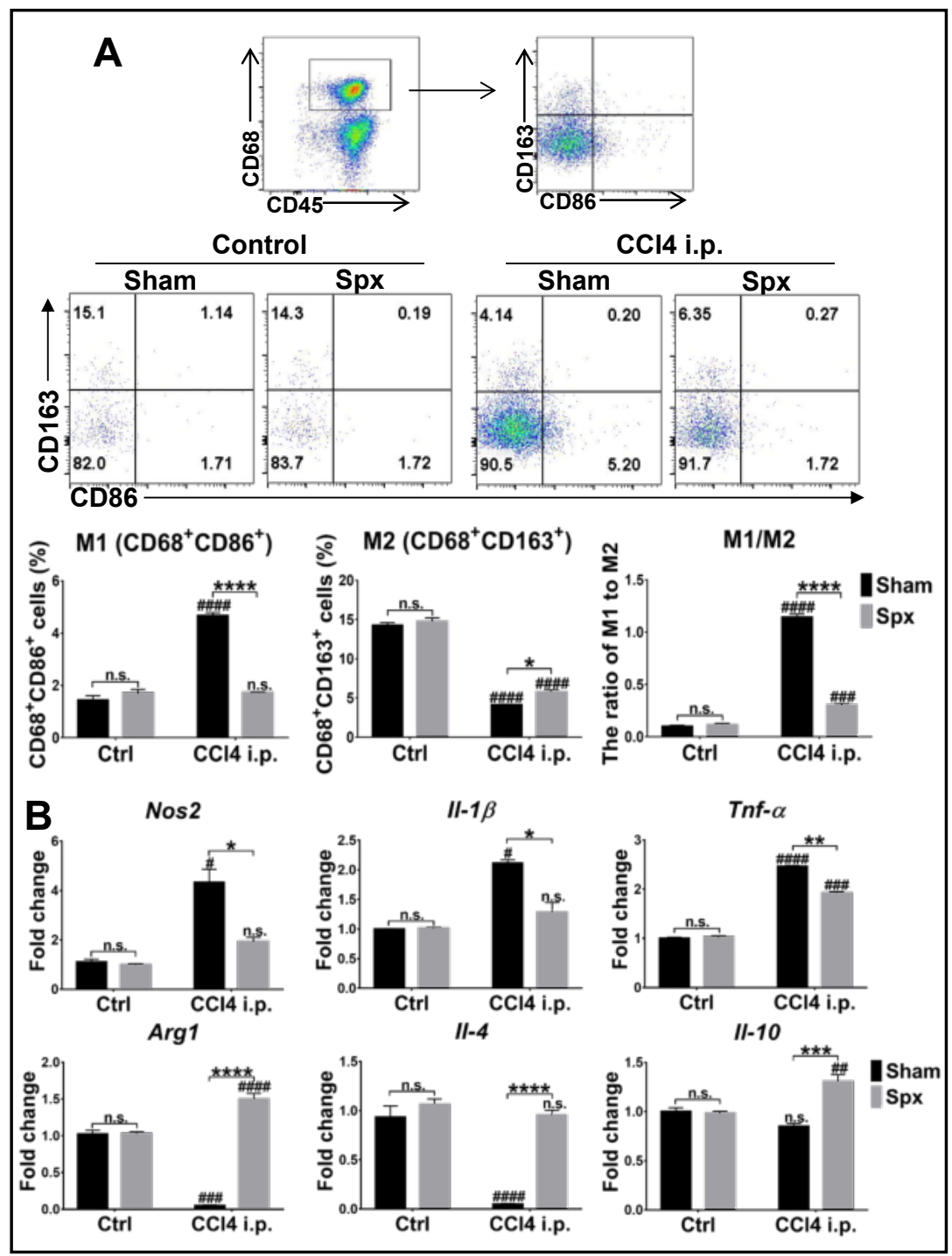

recipients and examined hepatic macrophages two weeks later (Supplementary Fig. 11C). Transfer of splenocytes from fibrotic donors increased the proportion of M1-like hepatic macrophages and slightly decreased the proportion of M2-like hepatic macrophages, thus increasing the M1/M2 ratio. Transfer of splenocytes from healthy controls partly increased the proportion of M1-like hepatic macrophages and the M1/M2 ratio but had no effect on the M2-like hepatic macrophage proportion (Fig. 6A). Adoptive transfer of splenocytes from fibrotic donors exacerbated the development of hepatic fibrosis compared with the adoptive transfer of splenocytes from healthy controls or PBS control (Fig. 6B). Taken together, these data confirm the pro-fibrotic effects of splenocytes isolated from a fibrotic host.

To determine whether CCL2-mediated monocyte infiltration was involved in the adoptive transfer-mediated changes in hepatic macrophage phenotype and liver fibrosis progression, we blocked CCL2 in vivo. First we examined the level of CCL2 in fibrotic livers post-transfer of different splenic cell types and found that only splenic macrophage transfer significantly increased CCL2 levels to an extent comparable to that of transferring total splenocytes. Transferring non-macrophage splenocytes did not alter hepatic levels of CCL2 (Supplementary Fig. 12A), confirming the central role of splenic macrophages in regulating CCL2 secretion in fibrotic liver. Next, we blocked CCL2 in vivo at $3 \mathrm{~h}$ after adoptive transfer of splenocytes. CCL2 blockade decreased the proportion of hepatic monocytes/macrophages 


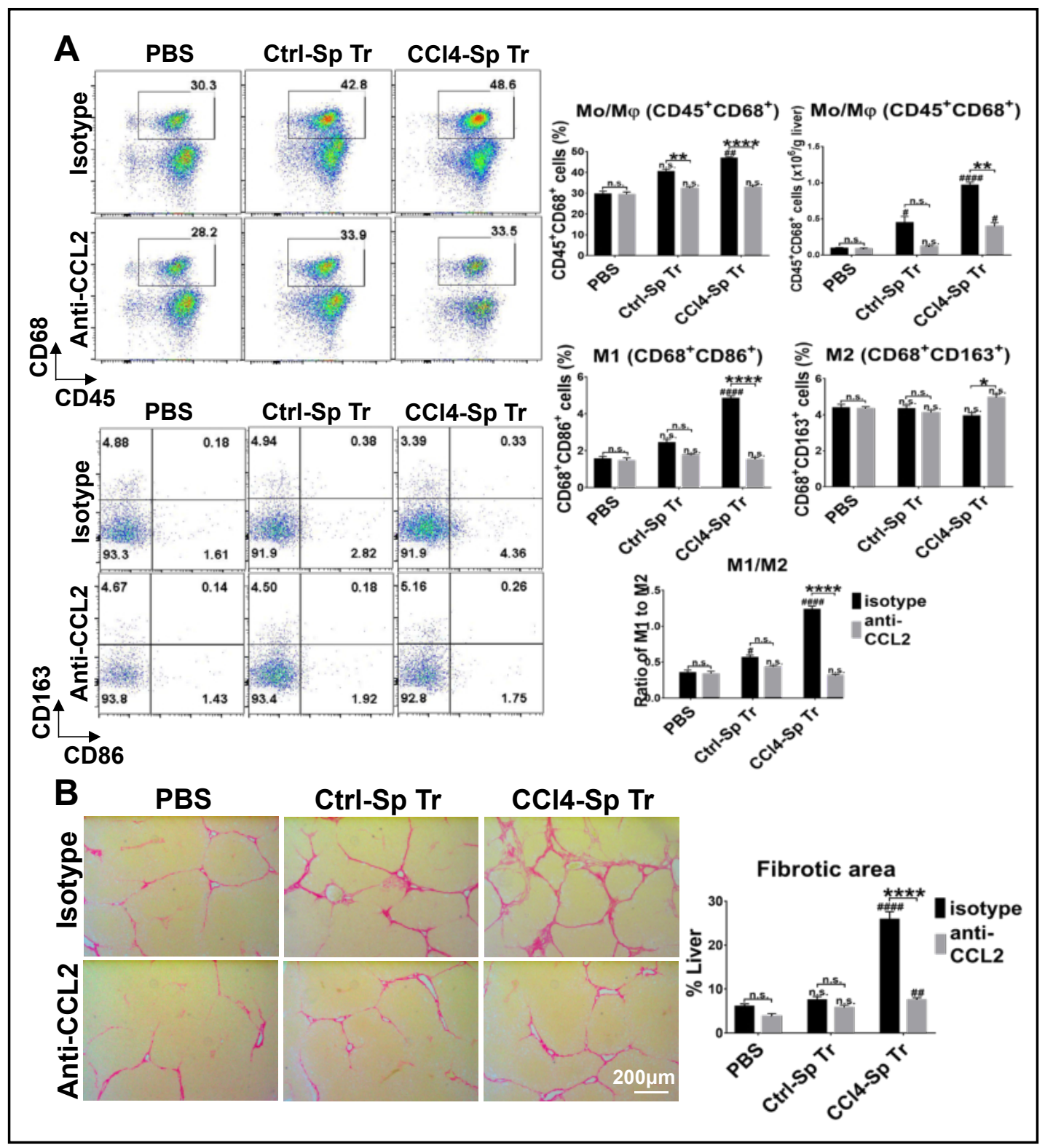

Fig. 6. Fibrotic donor splenocyte transfer to splenectomized recipients partially restores the M1-dominant hepatic macrophage phenotype and promotes fibrosis. (A) Carbon tetrachloride $\left(\mathrm{CCl}_{4}\right)$-induced fibrotic rats underwent splenectomy or sham operation $24 \mathrm{~h}$ after the eighth $\mathrm{CCl}_{4}$ injection, and received donor splenocytes with the administration of CCL2-neutralizing antibody or isotype control $72 \mathrm{~h}$ after Spx or sham operation, and euthanized $72 \mathrm{~h}$ after the last (12th) injection of $\mathrm{CCl}_{4}$. The percentages and absolute numbers of hepatic monocytes/macrophages $\left(\mathrm{CD} 45^{+} \mathrm{CD} 8^{+}\right)$were analyzed. The percentage of M1-like $\left(\mathrm{CD} 68^{+} \mathrm{CD} 6^{+}\right)$ and M2-like (CD68 $\left.{ }^{+} \mathrm{CD} 163^{+}\right)$macrophages and the ratio of M1 to M2 were determined. (B) Liver sections were stained with Sirius red and the proportion of fibrotic areas in liver sections quantified. Magnification: $\times 50$; scale bar: $200 \mu \mathrm{m}$. Statistics: two-way ANOVA ( $=5$ in each group). ${ }^{*} P<0.05,{ }^{* *} P<0.01,{ }^{* * *} P<0.001$,

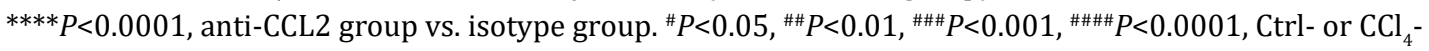
Sp Tr vs. PBS. Abbreviations: Sp-Tr, adoptive transfer of splenocytes; PBS, phosphate-buffered saline.

to almost PBS control levels and decreased the proportion of M1-like hepatic macrophages without altering the proportion of M2-like hepatic macrophages (Fig. 6A; Supplementary Fig. 12B). In addition, CCL2 blockade markedly reduced the amount of collagen deposition in injured liver (Fig. 6B; Supplementary Fig. 12B). Collectively, our data support a mechanism 


\section{Cellular Physiology Cell Physiol Biochem 2018;51:557-574 \begin{tabular}{ll|l} 
and Biochemistry Published onlıne: 20 November 2018 & $\begin{array}{l}\text { (c) } 2018 \text { The Author(s). Published by S. Karger AG, Basel } \\
\text { www.karger.com/cpb }\end{array}$ \\
\hline
\end{tabular} \\ Li et al.: The Spleen and Liver Fibrosis}

whereby splenic macrophages promote CCL2 production in hepatic macrophages and the subsequent CCL2-dependent expansion of M1-dominant hepatic macrophages and the exacerbation of liver fibrosis.

\section{Discussion}

Liver fibrosis is a highly conserved process in response to injury. Hepatic stellate cells are the major fibrogenic effectors in the injured liver, with hepatic macrophages coordinating pro-inflammatory and pro-fibrogenic signals. The spleen is the largest lymphoid organ and possesses highly elaborate tissue structures harboring multiple immune cell subsets. Splenic immune cells, particularly the resident macrophages and mobilizable monocytes within the reservoir in red pulp, are involved in various inflammatory diseases. Previously, the spleen was thought to promote the progression of liver fibrosis through the generation of pro-inflammatory and pro-fibrogenic factors [15]. However, whether and how the spleen can modulate hepatic monocytes/macrophages to affect liver fibrosis remains unclear.

In the present study, we have demonstrated that the spleen can promote hepatic macrophage secretion of CCL2 and contribute to the establishment of an M1-dominant macrophage phenotype during liver fibrosis. We found that splenectomy decreased the severity of hepatic fibrosis, and hepatic macrophage accumulation and CCL2 secretion, suggesting the splenic modulation of hepatic macrophage behavior during liver fibrosis. Ex vivo, we confirmed that splenic macrophages promoted CCL2 secretion via upregulation of SOCS3 signaling in hepatic macrophages. We further observed that splenocytes could migrate toward conditioned medium from hepatic macrophages isolated from fibrotic rats and that blockade of SOCS3-CCL2-CCR2 signaling weakened this migratory effect. In vivo, we observed that splenectomy could decrease the M1-dominant phenotype of hepatic macrophages and liver fibrosis severity while the adoptive transfer of splenocytes partially reversed these effects. CCL2 blockade after adoptive transfer of splenocytes restored the beneficial effects of splenectomy. Collectively, our data suggest that splenic macrophages can promote hepatic macrophage CCL2 secretion, which in turn facilitates monocyte recruitment and formation of an M1-dominant hepatic macrophage phenotype, and promotes liver fibrosis severity.

Previously, Yada et al. reported that splenectomy could affect hepatic macrophage phenotype and ameliorate collagen deposition in fibrotic mice [26], which is consistent with findings of our study. In their study, however, Yada and colleagues showed that hepatic macrophage numbers increased after splenectomy, which contrasts with our data. This discrepancy may be due to differences in the lifespan of the fibrosis model and splenectomy timepoint. In our study, we utilized a rat model of liver fibrosis induced by twice-weekly intraperitoneal injections of $\mathrm{CCl}_{4}$ for 6 consecutive weeks. Splenectomy or sham surgery was performed at the end of the fourth week of $\mathrm{CCl}_{4}$ administration. Thus, our study was performed at a relatively early stage in the development of liver fibrosis. In the study by Yada et al., liver fibrosis was induced by TAA (300 $\mathrm{mg} / \mathrm{L})$ in the drinking water given ad libitum for 32 weeks. Splenectomy or sham surgery was performed in the 32nd week, when the liver exhibited significant cirrhotic change and hypersplenism was observed. At this timepoint, the spleen may have also been fibrotic and hence capable of retaining or destroying circulating leukocytes [35], thereby contributing to leukocytopenia as demonstrated in their study [26]. Splenectomy therefore may have mitigated leukocytopenia and thus increased the degree of immune cell infiltration in the fibrotic liver. In our study, the spleen had not reached hypersplenism except for a slight and reactive enlargement (Supplementary Fig. 13). Thus, steady-state migration of leukocytes from the spleen would not have been impaired and may explain why splenectomy decreased hepatic macrophage accumulation in our study. Except for this discrepancy, both our findings and those of Yada et al. demonstrate that splenectomy can ameliorate fibrosis and decrease the M1/M2 macrophage ratio in fibrotic livers, emphasizing the role of the spleen in controlling the phenotype of hepatic macrophages and

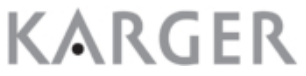


the progression of fibrosis.

Based on the finding that hepatic macrophage numbers in fibrotic rats were reduced following splenectomy, we hypothesized that the spleen may have contributed through modulation of monocyte recruitment into the liver. Since monocyte recruitment is a multistage process involving local chemokine release, cell migration, and extravasation or intravasation, it was possible that the spleen could regulate recruitment at any or even several of these stages. First we focused on the potential for splenic modulation of chemokine secretion in fibrotic liver. We found that both hepatic macrophages and stellate cells produced CCL2, with hepatic macrophages as the major source of CCL2, which is consistent with previous findings [36]. Hepatic macrophage CCL2 secretion was markedly reduced by splenectomy, reflecting splenic modulation of this process. Ex vivo, we confirmed that conditioned medium from splenic macrophages promoted CCL2 secretion by hepatic macrophages. Here, splenic macrophages referred to the resident macrophages of the spleen whilst the hepatic macrophage population would have comprised both resident Kupffer cells and recruited monocyte-derived macrophages (including splenic monocyte-derived macrophages). Due to the lack of available macrophage markers and the focused aim of our study, we were unable to differentiate between resident versus recruited hepatic macrophage subsets in fibrotic liver. Further investigations are needed to determine the potential differences between these macrophage subsets and their amenability to splenic modulation. In this study, we found that splenic macrophages modulated CCL2 secretion via upregulation of SOCS3 signaling in hepatic macrophages. The precise mechanism(s) controlling SOCS signaling in hepatic macrophages remains to be established. Considering that the spleen produces many soluble factors during liver fibrosis and these are hyperactivated, as demonstrated in our previous study [25], we hypothesize that the mechanism(s) may involve soluble factors such as TGF- $\beta$, TNF-a, and IL-6, or even exosomes released by splenic macrophages [37-40]. Macrophage secretion of CCL2 was inhibited by SOCS3 knockdown (Fig. 3). More importantly, bone marrow macrophages did not exert the same effects as splenic macrophages, further highlighting the key role of splenic macrophages in the modulation of the hepatic microenvironment.

SOCS proteins are regulators of inflammatory activity in macrophage. It has previously been reported that SOCS3 expression is increased during both acute and chronic inflammation [41]. Gordon et al. reported that SOCS3 siRNA-mediated gene silencing in M1-activated human monocyte-derived macrophages decreased the expression of proinflammatory factors [42]. Saeki et al. reported that cerulein-induced pancreatitis in mice involved the migration of $\mathrm{CD} 11 \mathrm{~b}^{\text {high }} \mathrm{CD} 11 \mathrm{c}-\mathrm{Gr}-1^{\text {low }}$ monocytes mediated by CCL2-CCR2 and SOCS3-dependent macrophage activation [33]. However, a direct association between SOCS3 signaling and CCL2 secretion has not been established. Our findings demonstrate that hepatic macrophage CCL2 secretion was SOCS3 dependent, thus highlighting the role of a local SOCS3-CCL2 axis in liver fibrosis.

In migration assays, we detected that the conditioned medium from hepatic macrophages could induce the migration of both bone marrow cells and splenocytes. Interestingly, however, blocking CCL2/CCR2 or attenuating SOCS3 expression affected the migration of only splenocytes (Fig. 4). These results suggest that crosstalk may exist between spleen and liver monocyte/macrophage subsets through selective chemokine/cytokine release, further highlighting the existence of a spleen-liver axis [16]. To our knowledge, our study is the first to highlight the interaction between splenic and hepatic macrophages via chemokine secretion and monocyte recruitment during liver fibrosis.

We identified an M1-dominant hepatic macrophage phenotype in vivo during liver fibrosis. It is well known that classical (M1-like) macrophages can act as pro-inflammatory effectors whilst alternatively activated (M2-like) macrophages facilitate the resolution of inflammation and tissue repair. Within fibrotic liver, there is most likely a spectrum of macrophage phenotypes with classical (M1-like) and alternative (M2-like) states representing two extremes [9]. We detected a marked decrease in M1-like and a slight increase in M2like hepatic macrophages following splenectomy, emphasizing a role for the spleen in shaping hepatic macrophage phenotypes. Since hepatic macrophages are key drivers of liver

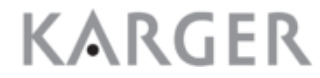




\section{Cellular Physiology Cell Physiol Biochem 2018;51:557-574 \begin{tabular}{ll|l} 
and Biochemistry & $\begin{array}{l}\text { DOl: 10.1159/000495276 } \\
\text { Published online: 20 November } 2018\end{array}$ & $\begin{array}{l}\text { 2018 The Author(s). Published by S. Karger AG, Basel } \\
\text { www.karger.com/cpb }\end{array}$ \\
\cline { 2 - 3 }
\end{tabular} \\ Li et al.: The Spleen and Liver Fibrosis}

fibrogenesis, our findings suggest that hepatic macrophage phenotype changes are another important downstream target of splenic modulation.

We also performed adoptive transfer studies to investigate whether the re-addition of splenocytes could reverse the protective effects induced by splenectomy. As hypothesized, we discovered that adoptive transfer of splenocytes could partly restore the M1-dominant hepatic macrophage phenotype and exacerbate liver fibrosis. More importantly, we also found that hepatic macrophage CCL2 production could be upregulated in vivo by adoptive transfer of splenic macrophages (Supplementary Fig. 11A), further confirming our initial ex vivo findings. Blocking CCL2 in vivo negated the pro-fibrogenic effects of splenocyte adoptive transfer (Fig. 6), highlighting the therapeutic value of targeting CCL2 during liver fibrosis. Theoretically, CCL2 blockade may attenuate the recruitment of both spleen-derived and bone marrow-derived monocytes (we cannot exclude the possibility that the latter has a contributory role in the therapeutic effect of CCL2 blockade). However, it is noteworthy that CCL2 blockade does negate the splenic contribution to hepatic fibrogenesis. Further investigation is required to determine the functions and potential pathologic contributions of spleen-derived compared with bone marrow-derived monocytes during liver fibrosis.

Our findings point to a pathological role for the spleen during liver fibrosis. The results of a recent study by Aoyama et al. using a $\mathrm{CCl}_{4}$-induced mouse model suggested that the spleen may also play a protective role in liver fibrosis through production of a key antimicrobial protein Lipocalin-2, which is an important regulator of Kupffer cell activation and gut-liver interaction [43]. This contradictory conclusion may be due to splenectomy being performed prior to $\mathrm{CCl}_{4}$ injection in their study. Overall, when considering our findings together with those of Aoyama et al. and Yada et al., there is a possibility that the pathological, or even protective, effects of the spleen in liver fibrosis are critically dependent on time or disease stage. This possibility would most likely be due to different histological and cellular alterations manifesting in the spleen throughout the different stages of liver fibrosis, and will be an important focus for future study.

\section{Conclusion}

We demonstrated that splenic macrophages promote hepatic macrophage CCL2 secretion, which in turn facilitates monocyte recruitment and the establishment of an M1dominant hepatic macrophage phenotype and the augmentation of liver fibrosis.

\section{Acknowledgements}

This work was supported by the National Natural Science Foundation of China (No. 91442122, No. 81600100) and the Program for Changjiang Scholars and Innovative Research Team in University (No. IRT1171). We thank Wei Wei, Zhenzhen Li, Haiyan Chen, Yu Li, Wei Jiang and Weisan Chen for their contributions to this study.

\section{Disclosure Statement}

The authors declare that no conflicts of interest exists.

\section{References}

1 Bataller R, Brenner DA: Liver fibrosis. Journal Of Clinical Investigation 2005; 115:209-218.

-2 Pellicoro A, Ramachandran P, Iredale JP, Fallowfield JA: Liver fibrosis and repair: immune regulation of wound healing in a solid organ. Nature Reviews Immunology 2014;14:181-194. 


\section{Cellular Physiology Cell Physiol Biochem 2018;51:557-574 and Biochemistry \begin{tabular}{l|l} 
DOI: 10.1159/000495276 2018 The Author(s). Published by S. Karger AG, Basel \\
(c)
\end{tabular}

3 Krenkel 0, Tacke F: Liver macrophages in tissue homeostasis and disease. Nature Reviews Immunology 2017;17:306-321.

4 Karlmark KR, Weiskirchen R, Zimmermann HW, Gasssler N, Ginhoux F, Weber C, Merad M, Luedde T, Trautwein C, Tacke F: Hepatic Recruitment of the Inflammatory Gr1+ Monocyte Subset Upon Liver Injury Promotes Hepatic Fibrosis. Hepatology 2009;50:261-274.

5 McDonald B, Kubes P: Innate Immune Cell Trafficking and Function During Sterile Inflammation of the Liver. Gastroenterology 2016;151:1087-1095.

-6 Tacke F: Targeting hepatic macrophages to treat liver diseases. Journal Of Hepatology 2017;66:1300-1312.

$>7$ Murray PJ, Wynn TA: Protective and pathogenic functions of macrophage subsets. Nature Reviews Immunology 2011;11:723-737.

-8 Sica A, Invernizzi P, Mantovani A: Macrophage Plasticity and Polarization in Liver Homeostasis and Pathology. Hepatology 2014;59:2034-2042.

-9 Okada K, Arai S, Itoh H, Adachi S, Hayashida M, Nakase H, Ikemoto M: CD68 on rat macrophages binds tightly to S100A8 and S100A9 and helps to regulate the cells' immune functions. Journal Of Leukocyte Biology 2016;100:1093-1104.

10 Ma SF, Chen YJ, Zhang JX, Shen L, Wang R, Zhou JS, Hu JG, Lu HZ: Adoptive transfer of M2 macrophages promotes locomotor recovery in adult rats after spinal cord injury. Brain Behavior And Immunity 2015;45:157-170.

11 Bronte V, Pittet MJ: The Spleen in Local and Systemic Regulation of Immunity. Immunity 2013;39:806-818.

12 Swirski FK, Nahrendorf M, Etzrodt M, Wildgruber M, Cortez-Retamozo V, Panizzi P, Figueiredo JL, Kohler RH, Chudnovskiy A, Waterman P, Aikawa E, Mempel TR, Libby P, Weissleder R, Pittet MJ: Identification of Splenic Reservoir Monocytes and Their Deployment to Inflammatory Sites. Science 2009;325:612-616.

13 Weller S, Braun MC, Tan BK, Rosenwald A, Cordier C, Conley ME, Plebani A, Kumararatne DS, Bonnet D, Tournilhac O, Tchernia G, Steiniger B, Staudt LM, Casanova JL, Reynaud CA, Weill JC: Human blood IgM "memory" B cells are circulating splenic marginal zone B cells harboring a prediversified immunoglobulin repertoire. Blood 2004;104:3647-3654.

14 Wu L, Parekh VV, Hsiao J, Kitamura D, Van Kaer L: Spleen supports a pool of innate-like B cells in white adipose tissue that protects against obesity-associated insulin resistance. Proceedings Of the National Academy Of Sciences Of the United States Of America 2014;111:E4638-E4647.

15 Li LA, Duan MB, Chen WS, Jiang A, Li XM, Yang J, Li ZF: The spleen in liver cirrhosis: revisiting an old enemy with novel targets. Journal Of Translational Medicine 2017;15:

16 Tarantino G, Scalera A, Finelli C: Liver-spleen axis: Intersection between immunity, infections and metabolism. World Journal Of Gastroenterology 2013;19:3534-3542.

17 Oberti F, Valsesia E, Pilette C, Rousselet MC, Bedossa P, Aube C, Gallois Y, Rifflet H, Maiga MY, PenneauFontbonne D, Cales P: Noninvasive diagnosis of hepatic fibrosis or cirrhosis. Gastroenterology 1997;113:1609-1616.

18 Kisseleva T, Uchinami H, Feirt N, Quintana-Bustamante O, Segovia JC, Schwabe RF, Brenner DA: Bone marrow-derived fibrocytes participate in pathogenesis of liver fibrosis. Journal Of Hepatology 2006;45:429-438.

19 Murata K, Ito K, Yoneda K, Shiraki K, Sakurai H, Ito M: Splenectomy improves liver function in patients with liver cirrhosis. Hepato-Gastroenterology 2008;55:1407-1411.

$\checkmark 20$ Akahoshi T, Hashizume M, Tanoue K, Shimabukuro R, Gotoh N, Tomikawa M, Sugimachi K: Role of the spleen in liver fibrosis in rats may be mediated by transforming growth factor beta-1. Journal of Gastroenterology And Hepatology 2002;17:59-65.

21 Li ZF, Zhang Y, Gao J, Zhang PJ, Wang JX, Liu XG: [Expression and significance of Toll-like receptor 4 of splenic macrophage in patients with hypersplenism due to portal hypertension]. Zhonghua Yi Xue Za Zhi 2004;84:1088-1091.

22 Li Z, Zhang S, Huang C, Zhang W, Hu Y, Wei B: MicroRNAome of splenic macrophages in hypersplenism due to portal hypertension in hepatitis B virus-related cirrhosis. Exp Biol Med (Maywood) 2008;233:14541461.

23 Li A, Li Z, Ma S, Su Q, Zhang S, Sun X, Li G: Dysfunction of splenic macrophages in cirrhotic patients with hypersplenism and HBV infection. Am J Med Sci 2008;336:32-38. 


\section{Cellular Physiology Cell Physiol Biochem 2018;51:557-574

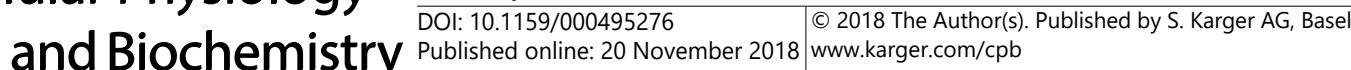

24 Jiang A, Zhang S, Li Z, Liang R, Ren S, Li J, Pu Y, Yang J: miR-615-3p promotes the phagocytic capacity of splenic macrophages by targeting ligand-dependent nuclear receptor corepressor in cirrhosis-related portal hypertension. Exp Biol Med (Maywood) 2011;236:672-680.

25 Ren S, Zhang S, Li M, Huang C, Liang R, Jiang A, Guo Y, Pu Y, Huang N, Yang J, Li Z: NF-kappaB p65 and c-Rel subunits promote phagocytosis and cytokine secretion by splenic macrophages in cirrhotic patients with hypersplenism. Int J Biochem Cell Biol 2013;45:335-343.

-26 Yada A, Iimuro Y, Uyama N, Uda Y, Okada T, Fujimoto J: Splenectomy attenuates murine liver fibrosis with hypersplenism stimulating hepatic accumulation of Ly-6C(lo) macrophages. J Hepatol 2015;63:905-916.

27 Iredale JP, Benyon RC, Pickering J, McCullen M, Northrop M, Pawley S, Hovell C, Arthur MJ: Mechanisms of spontaneous resolution of rat liver fibrosis. Hepatic stellate cell apoptosis and reduced hepatic expression of metalloproteinase inhibitors. J Clin Invest 1998;102:538-549.

28 He F, Guo FC, Li Z, Yu HC, Ma PF, Zhao JL, Feng L, Li WN, Liu XW, Qin HY, Dou KF, Han H: Myeloid-specific disruption of recombination signal binding protein Jkappa ameliorates hepatic fibrosis by attenuating inflammation through cylindromatosis in mice. Hepatology 2015;61:303-314.

29 Weiskirchen R, Gressner AM: Isolation and culture of hepatic stellate cells. Methods Mol Med 2005;117:99113.

-30 Yang L, Kwon J, Popov Y, Gajdos GB, Ordog T, Brekken RA, Mukhopadhyay D, Schuppan D, Bi Y, Simonetto D, Shah VH: Vascular endothelial growth factor promotes fibrosis resolution and repair in mice. Gastroenterology 2014;146:1339-1350 e1331.

-31 Nielsen SR, Quaranta V, Linford A, Emeagi P, Rainer C, Santos A, Ireland L, Sakai T, Sakai K, Kim YS, Engle D, Campbell F, Palmer D, Ko JH, Tuveson DA, Hirsch E, Mielgo A, Schmid MC: Macrophage-secreted granulin supports pancreatic cancer metastasis by inducing liver fibrosis. Nat Cell Biol 2016;18:549-560.

-32 Thacker MA, Clark AK, Bishop T, Grist J, Yip PK, Moon LD, Thompson SW, Marchand F, McMahon SB: CCL2 is a key mediator of microglia activation in neuropathic pain states. Eur J Pain 2009;13:263-272.

-33 Saeki K, Kanai T, Nakano M, Nakamura Y, Miyata N, Sujino T, Yamagishi Y, Ebinuma H, Takaishi H, Ono Y, Takeda K, Hozawa S, Yoshimura A, Hibi T: CCL2-induced migration and SOCS3-mediated activation of macrophages are involved in cerulein-induced pancreatitis in mice. Gastroenterology 2012;142:1010-1020 e1019.

-34 Martin-Fernandez B, Rubio-Navarro A, Cortegano I, Ballesteros S, Alia M, Cannata-Ortiz P, Olivares-Alvaro E, Egido J, de Andres B, Gaspar ML, de Las Heras N, Lahera V, Moreno JA: Aldosterone Induces Renal Fibrosis and Inflammatory M1-Macrophage Subtype via Mineralocorticoid Receptor in Rats. PLoS One 2016;11:e0145946.

35 Stefanescu H, Grigorescu M, Lupsor M, Procopet B, Maniu A, Badea R: Spleen stiffness measurement using Fibroscan for the noninvasive assessment of esophageal varices in liver cirrhosis patients. J Gastroenterol Hepatol 2011;26:164-170.

-36 Marra F, Tacke F: Roles for chemokines in liver disease. Gastroenterology 2014;147:577-594 e571.

-37 Lovibond AC, Haque SJ, Chambers TJ, Fox SW: TGF-beta-induced SOCS3 expression augments TNF-alphainduced osteoclast formation. Biochem Biophys Res Commun 2003;309:762-767.

-38 Sha K, Yeh S, Chang C, Nastiuk KL, Krolewski JJ: TNF signaling mediates an enzalutamide-induced metastatic phenotype of prostate cancer and microenvironment cell co-cultures. Oncotarget 2015;6:25726-25740.

-39 Carow B, Rottenberg ME: SOCS3, a Major Regulator of Infection and Inflammation. Front Immunol 2014;5:58.

-40 Bourdonnay E, Zaslona Z, Penke LR, Speth JM, Schneider DJ, Przybranowski S, Swanson JA, Mancuso P, Freeman CM, Curtis JL, Peters-Golden M: Transcellular delivery of vesicular SOCS proteins from macrophages to epithelial cells blunts inflammatory signaling. J Exp Med 2015;212:729-742.

41 White GE, Cotterill A, Addley MR, Soilleux EJ, Greaves DR: Suppressor of cytokine signalling protein SOCS3 expression is increased at sites of acute and chronic inflammation. J Mol Histol 2011;42:137-151.

42 Gordon P, Okai B, Hoare JI, Erwig LP, Wilson HM: SOCS3 is a modulator of human macrophage phagocytosis. J Leukoc Biol 2016;100:771-780.

43 Aoyama T, Kuwahara-Arai K, Uchiyama A, Kon K, Okubo H, Yamashina S, Ikejima K, Kokubu S, Miyazaki A, Watanabe S: Spleen-derived lipocalin-2 in the portal vein regulates Kupffer cells activation and attenuates the development of liver fibrosis in mice. Lab Invest 2017;97:890-902. 